\title{
A severe wind hazard model for non-cyclonic regions of Australia using simulated climate data
}

\author{
L.A. Sanabria , C.M. Thomas and R.P. Cechet \\ Environmental Geoscience Division, Geoscience Australia, Canberra, Australia \\ Email:augusto.sanabria@ga.gov.au
}

\begin{abstract}
Severe wind is one of the major natural hazards affecting Australia. The main wind hazards contributing to economic loss in Australia are tropical cyclones, thunderstorms and mid-latitude storms. Geoscience Australia's Environmental Geoscience Division (EGD) has developed mathematical models to study a number of natural hazards including wind hazard.

In this paper we describe a methodology to study "combined" gust wind hazard produced by thunderstorm and mid-latitude or synoptic storms. The methodology is aimed at applications in regions where these two wind types dominate the hazard spectrum across all return periods (most of the Australian continent apart from the coastal region stretching north from about $27^{\circ} \mathrm{S}$ where the hazard for large return periods is dominated by tropical cyclones). Each of these severe wind types is generated by different physical phenomena and poses a different hazard to the built environment. For these reasons, it is necessary to model them separately. The return period calculated for each wind type is then combined probabilistically to produce the combined gust wind return period, the indicator used to quantify severe wind hazard.
\end{abstract}

The combined wind hazard model utilises climate model simulated wind speeds and hence it allows wind analysts to assess the impact of climate change on future wind hazard. It aims to study severe wind hazard in the non-cyclonic regions of Australia which is the region ' $A$ ', as defined in the Australian/NZ Wind Loading Standard (AS/NZS 1170.2:2011). This region is dominated by thunderstorm and synoptic winds.

The synoptic wind component of the combined wind hazard comprises three sub-models:

- A technique to extract and process mean wind speeds from a high-resolution regional climate model.

The climate model used in these studies provides maximum time-step wind speeds each hour at a resolution of $14 \mathrm{~km}$, which does not resolve thunderstorms or tropical cyclones.

- A Monte Carlo method to generate gust wind speeds from the climate-modelled mean wind speeds; and

- A statistical model to quantify wind hazard in terms of return period using extreme value distributions.

The analysis of the thunderstorm wind hazard is made using the observational data from a number of Bureau of Meteorology (BoM) recording stations located around the country. Thunderstorm wind speeds are extracted by matching BoM weather description and wind speed datasets. An extreme value analysis is made on this dataset and the parameters of the resulting Generalised Pareto Distributions are interpolated spatially. This technique can be used to assess thunderstorm hazard under current climate conditions. For future climate, the model extracts two parameters from the regional climate model: the Convective Available Potential Energy (CAPE) and wind speed (surface and vertical profile). Using these two parameters it is possible to make an assessment of the likelihood of the formation of severe thunderstorms. The model allows estimation of possible changes in thunderstorm frequency and hence an assessment of thunderstorm wind hazard under future climate conditions, under the assumption that the intensity of thunderstorm downbursts remains constant.

To illustrate the methodology, severe wind hazard calculations under current and future climate conditions for the Australian state of Tasmania will be presented. The results show increases particularly in the regions which currently have high hazard. This increase is however not uniform across the state and is more apparent in the later part of the $21^{\text {st }}$ century (greater greenhouse gas emissions forcing) than in the early part. Implications of this projected change for building standards will be discussed.

Keywords: Wind hazard, regional climate models, mean and gust wind speed. 


\section{INTRODUCTION}

Following the release of the report on natural disasters in Australia by the Department of Transport and Regional Services (DOTARS, 2004) Geoscience Australia (GA) has been developing risk models to assess the potential losses to Australian communities from a range of sudden impact natural hazards (Middelmann, 2007). Severe wind is one of the major hazards facing Australia. While cyclonic winds are the major source of wind hazard in the northern states, non-cyclonic winds driven by synoptic lows, thunderstorms and tornadoes affect the southern states. Severe winds were responsible for about $40 \%$ of damage to Australian residential buildings during the $20^{\text {th }}$ century (Chen, 2004).

This paper describes a methodology to assess severe wind hazard in the non-cyclonic regions of Australia, region 'A', as defined in the Australian/NZ Wind Loading Standard (AS/NZS 1170.2:2011), which are dominated by thunderstorm and synoptic winds. Since these two phenomena are generated by different physical mechanisms, occurring at different spatio-temporal scales and pose a different hazard to the built environment it is important to model them separately. For these reasons the methodology discussed here comprises two components: a synoptic wind hazard model and a thunderstorm wind hazard model.

The models use both observational and climate model simulated wind speed data. The use of climate model simulated data allows wind analysts to assess wind hazard over an entire region and, more importantly, to consider the impact of climate change on future wind hazard.

\section{MODEL DESCRIPTION}

\subsection{Synoptic wind component}

The synoptic wind model of the combined wind hazard assessment comprises three sub-models:

- A statistical model to quantify wind hazard in terms of return period using extreme value distributions;

- A technique to extract and process mean wind speeds from a high-resolution regional climate model; and

- A Monte Carlo method to generate gust wind speeds from the climate-modelled mean wind speeds.

The core of the statistical model is the fitting of Extreme Value (EV) distributions to a dataset of wind speeds in order to calculate gust wind hazard. To do this the model calculates the return period of maximum daily gust wind speed using the Generalised Pareto Distribution (GPD) (Coles, 2001; Sanabria and Cechet, 2007).

The climate simulation data used for this project were generated by the Climate Futures for Tasmania project (CFT). The CFT team dynamically downscaled six general circulation models using CSIRO's Conformal Cubic Atmospheric Model (CCAM) (Corney et al., 2010). Simulations focusing on Tasmania, using IPCC scenarios B1 and A2, for the period 1960 to 2100, were considered. Hourly mean wind speeds (10 meter height over open terrain) were produced from each of the six ensemble members. The mean hourly speeds were then transformed to maximum daily mean speeds. CCAM was also utilised to downscale the NCEP reanalysis data (1961-2000), the results of which will be used in Section 2.2.

In wind hazard studies the variable of interest is gust wind speed so a Monte Carlo-based (MC) method to generate gust winds from the climate model simulated mean wind speeds was developed. The MC method works by simulating the physics of wind generation for synoptic wind conditions. The process consists of the numerical convolution of the mean wind speed and an empirical gust factor (GF) to produce gust wind speeds (Sanabria and Cechet, 2010). The gust factor is defined as the ratio of maximum wind speed (gust) and mean wind speed for the same time period (generally ten minutes duration). For this study the GF was calculated from the half-hourly observational wind speed datasets provided by the Bureau of Meteorology (BoM), which contain the maximum gust and mean wind speed for the last 10 minutes of each period.

In order to capture the regional characteristics of Tasmania, observed wind speed data for Hobart, Launceston and Wynyard recording stations were acquired from BoM. These sites were selected because their weather stations and anemometer measurements are located at airports, avoiding the problem of the urban environment or trees affecting instrumentation, and also because these stations having gust wind speed records. The three datasets were joined into a single super-station in order to calculate a regional GF, which was applied to all of Tasmania. This GF was used for the sampling process in the Monte Carlo simulation. To correct the bias which exists when comparing area-averaged climate simulated and location-based wind speeds, the return period (RP) of the observed gust wind speeds at the three selected stations were generated and a bias correction factor (ratio of observed RP and CCAM-generated RP of current climate) was calculated. This forces the CCAM-generated RP of gust winds at the selected stations to be similar to the observed. This correction factor was applied to all cells in the grid. 


\subsection{Thunderstorm wind component}

Development of a model to study thunderstorm hazard is complicated by the fact that climate model simulations do not resolve thunderstorms. These are small-scale phenomena with distance scales of the order of kilometers and time scales of the order of one hour. Regardless of whether models existed to capture these phenomena they would need to be run at high spatial and temporal resolution and their application over large areas and for times long enough to provide sufficient data for statistical analysis would not be possible with current computing resources.

A series of models published in recent years were assessed for this project but their results were judged to be too poor for practical applications (Thomas and Arthur, 2011). For this reason it was decided to develop a two-part model, the first part calculates wind hazard for current climate using observational data and the second part calculates wind hazard for future climate conditions using high resolution climate-simulated data to adjust the frequency of thunderstorm occurrence under the assumption that thunderstorm intensity does not change (since there is no way to model intensity under future climate scenarios).

The thunderstorm model was developed for mainland Australia and extended later to Tasmania. For the current climate the thunderstorm model utilises three datasets provided by BoM: maximum daily gust wind speeds (3 second average); a weather description dataset; and an historical cyclone tracks database. Initially the model extracts thunderstorm wind speeds from 56 BoM stations scattered throughout Australia (Thomas and Arthur, 2011). The extraction is based on the merging of the weather description and maximum daily gust wind speed datasets. The weather description dataset contains a description of the weather conditions at the station at three-hour intervals. The data describe both the present weather conditions (past hour) and the past weather using a set of 100 symbols representing various weather conditions. Seventeen of these symbols are taken to represent thunderstorms, and in this way the maximum daily winds that may be attributed to thunderstorms can be extracted from the merged dataset. This procedure distinguishes between thunderstorm and non-thunderstorm maximum daily gusts, but does not characterize cyclonic winds. Furthermore, there are a large number of records in the merged dataset that have no associated weather description. To address these problems the merged dataset is matched with the historical cyclone track database to further classify the gust wind speeds as follows:

- If the record contains one of the 17 symbols characterizing thunderstorms in either the present or past weather description then it is classified as a thunderstorm;

- Any record not classified as a thunderstorm, and which is within 12 hours and $75 \mathrm{~km}$ of a record in the cyclone track database is classified as a cyclonic gust;

- Gusts which have associated weather descriptions and which are not classified as thunderstorm or cyclonic winds using the above procedure are classified as synoptic gusts and their maximum speed is found; and

- $\quad$ Any unclassified gusts with a speed exceeding this maximum are classified as thunderstorms.

In the final step the model fits a GPD to the extracted thunderstorm dataset at each of the BoM stations. This gives the exceedance level $w$ for a given return period $t$ (in years) as:

$$
w(t)=u+\frac{\sigma}{\xi}\left[(365.25 t \varsigma)^{\xi}-1\right]
$$

where $u, \sigma, \xi, \zeta$ are respectively the threshold, scale, shape and probability of exceedance of the generalized Pareto distributions. They are different for each of the BoM stations.

To obtain exceedance levels at other locations the quantities $\sigma, \xi$ and $\zeta$ are standardized to refer to a single value of the threshold $u$, and are interpolated to a grid using an inverse distance weighted algorithm. The exceedance levels for each cell of the grid can then be calculated at various return periods using the interpolated GPD parameters (Thomas and Arthur, 2011). The inverse distance weighted algorithm employed here should be regarded as a very crude first step in this direction, and there are more sophisticated techniques that should be investigated.

Thunderstorm hazard under future climate conditions was assessed by considering the environmental atmospheric conditions that contribute to the formation of thunderstorms making it possible to assess changes in thunderstorm frequency. Several studies have shown that the Convective Available Potential Energy (CAPE) and wind shear can be used to identify environmental conditions that are likely to produce thunderstorms. In particular Trapp et al. (2007) have found that the criterion 
Sanabria et al., A severe wind hazard model for non-cyclonic regions using climate-simulated data

$C A P E \times S_{6} \geq 10000$

with the additional conditions $C A P E \geq 100 \mathrm{~J} / \mathrm{Kg},\left|\boldsymbol{V}_{6}\right| \geq\left|\boldsymbol{V}_{o}\right|$ and $S_{6} \geq 5 \mathrm{~m} / \mathrm{s}$

can be used to distinguish environments that are likely to produce severe thunderstorms, and have used this to make estimates of the increase in severe thunderstorm frequency under future climate conditions by using output from climate models to estimate the frequency at which this criterion is met.

Here: $\quad \boldsymbol{V}_{6}=$ wind speeds at six $\mathrm{km}$ above ground; $\boldsymbol{V}_{o}=$ wind speeds at the surface level; $S_{6}=\left|\boldsymbol{V}_{6}-\boldsymbol{V}_{o}\right|$

For the case of Tasmania the NCEP reanalysis data for the period 1961 to 1990, and the output from various global circulation models, was downscaled using CCAM (see Section 2.1), and the resulting datasets used to compute the annual probability of the Trapp criterion being met on a pixel by pixel basis, both under current climate and future climate scenarios. If thunderstorm intensity is assumed to remain constant over a region, or a period of time, then variations in thunderstorm hazard are attributable to changes in storm frequency alone. In terms of Equation (1), this amounts to variations in $\zeta$, while holding the other parameters constant. This variation can be applied spatially as well as temporally.

In the case of Tasmania, the observations from the BoM stations at Hobart and Launceston airports were combined, while those from Wynyard airport (not staffed by a BoM trained observer) were discarded as being too unreliable. This resulted in values of $u=13.3, \sigma=5.847, \xi=-0.208$ and $\zeta=0.005353$. Since it was not possible to obtain spatially varying estimates of the distribution parameters from just a single location, the values of $u, \sigma$ and $\xi$ were assumed to remain constant over the state, corresponding to the assumption that thunderstorm intensity is spatially constant, and the value of $\zeta$ was varied spatially according to the formula:

$$
\varsigma_{\text {cell }}=\frac{\text { NCEP Trapp criterion exceedance frequency at givencell }}{\text { NCEPTrapp criterion exceedancefrequency at Hobart }} \times 5.353 \times 10^{-3}
$$

Applying this in Equation (1) gives an estimate of the exceedance levels at various return periods for current climate conditions over the state.

A similar procedure was used for the assessment of changes in thunderstorm hazard under future climate scenarios, though in this case the value of $\zeta$ was varied temporally. Ensemble means of Trapp exceedance frequencies for various periods under future climate scenarios were compared with the exceedance frequencies for a reference period (1961-1990) and the current climate probabilities, $\zeta$, were varied according to the ratio. This operation was performed over the whole grid. Full details are given in Thomas and Arthur (2011).

\section{RESULTS}

The methodology was applied to the Tasmanian region to calculate RPs of combined gust wind speeds. Figure 1 shows a map of the Tasmanian elevation contour.

\subsection{Current climate}

\section{Synoptic and thunderstorm wind hazard}

For each grid cell, mean wind speeds were extracted from the CCAM simulations (using the SRES A2 emissions scenario) for the period 1961 to 1990 . Then representative synoptic gust wind speeds in each cell were generated using the MC process outlined in Section 2.1. From these gust speeds, exceedance levels for 50 to 2000 year RP were calculated using the statistical model. Figure 2 shows the six model average (ensemble average) 500-year RP of synoptic gust wind speeds for current climate. As expected, they follow closely the features of Tasmanian topography, in particular note the increase of synoptic wind speeds due to the effect of the mountain slope in both the west and the north-east parts of Tasmania. This is consistent with detailed studies of the effect of mountain slopes on gust wind speeds (Holmes, 2007). Figure 3 shows the corresponding six model average 500-year return period of thunderstorm wind hazard generated using the procedure described in Section 2.2. Figure 3 shows that changes in thunderstorm frequency using this procedure do not result in large changes in hazard as discussed before (that is why a reduced colourbar graduation has been used in Figure 3 in order to show these changes, otherwise they will not be apparent). 


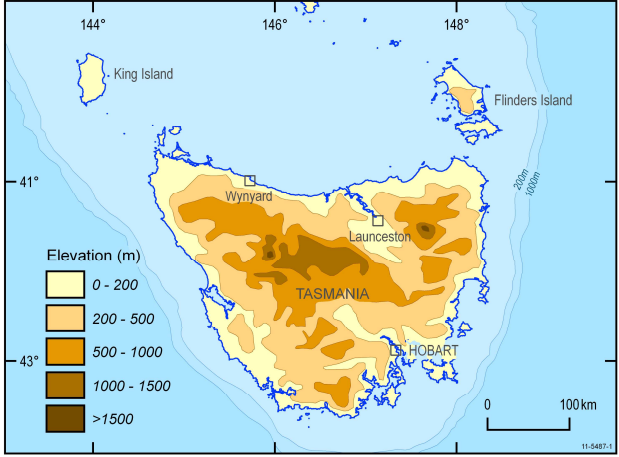

Figure 1. Contour map of Tasmanian elevation

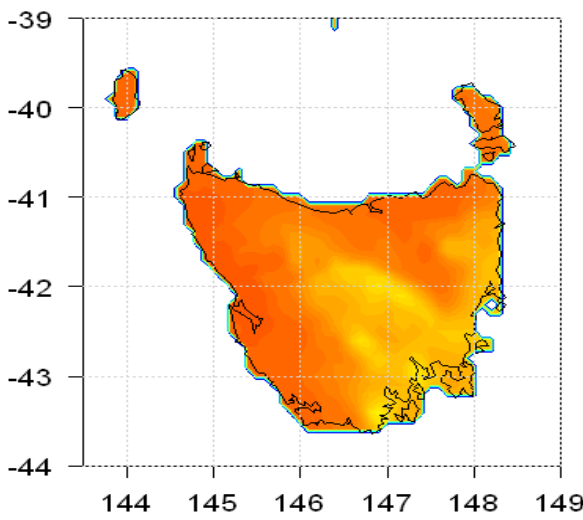

$\mathbf{m} / \mathbf{s}$

Figure 3. Average 500-yr RP of thunderstorm gust wind for current climate based on simulations from the six models.

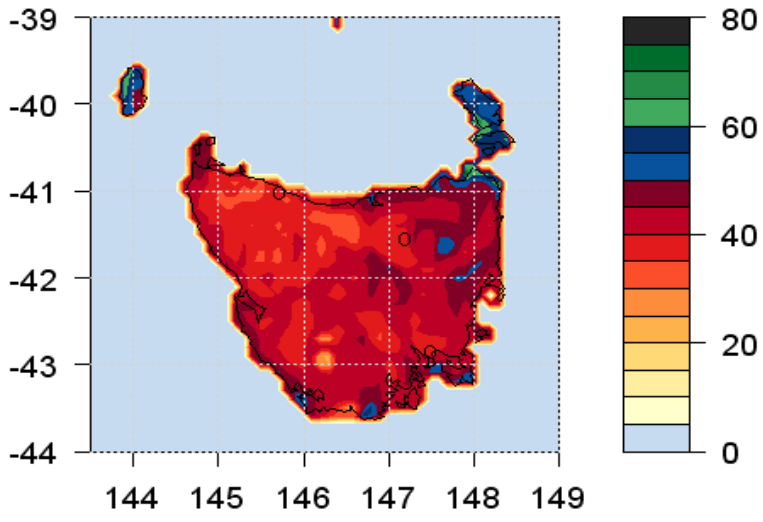

Figure 2. Average 500-yr RP of synoptic gust wind speeds for current climate (1961-1990) based on simulations from the six models.
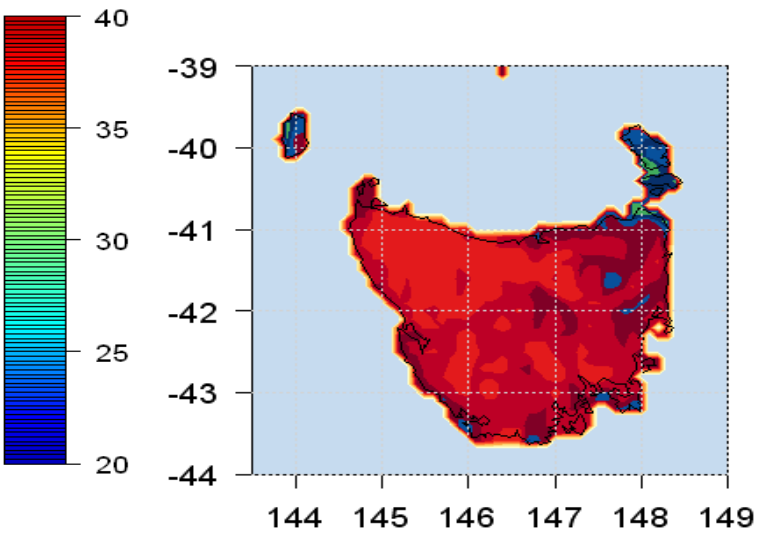

$\mathbf{m} / \mathbf{s}$

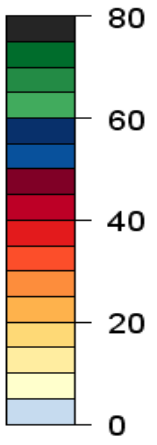

Figure 4. Average 500-yr RP of combined gust wind for current climate based on simulations from the six models.

Figure 4 presents the combined 500-yr RP of gust wind speed, obtained by combining probabilistically the return periods of Figures 2 and 3 (Holmes, 2007).

\subsection{Future climate}

To observe the wind hazard trend under climate change conditions, the procedure described above was applied to CCAM data extracted from a 20-year window between 2080 and 2100. Figure 5 shows the six model average 500-yr RP of combined gust wind speed for 2080-2100. Comparing Figures 4 and 5 it is possible to distinguish an increase in wind hazard by 2080-2100 with respect to current climate, an increase which is however not uniform across the Tasmanian region. This difference is more clearly distinguished in Figure 6 which depicts the percentage difference between 2080-2100 (A2 scenario) and current climate. A substantial increase in wind hazard by 2080-2100 is shown in the regions which currently have a high hazard (north-east, north-west and Bass Strait Islands), and a smaller increase in the south and central areas of the State is observed. To observe the difference in wind hazard when the B1 scenario (a low greenhouse gas emissions scenario) is used Figure 7 shows the percentage difference between the A2 and B1 scenarios for the (2080-2100) time window as a percentage of the B1 scenario. Figure 7 shows many spatial similarities when compared with Figure 6. The increase in hazard when using a higher emissions scenario (A2) results in an increase in wind hazard in the regions which currently have high hazard (north-east and north-west) and also in the south-west. This difference in the increase between the two scenarios is much lower than the increase of hazard presented in Figure 6 (for this reason a reduced colourbar graduation has to be used in 
Figure 6 otherwise the difference will not be visible) i.e. the hazard increase by the end of the century appears to be a function of the $\mathrm{CO} 2$ emissions in the atmosphere.

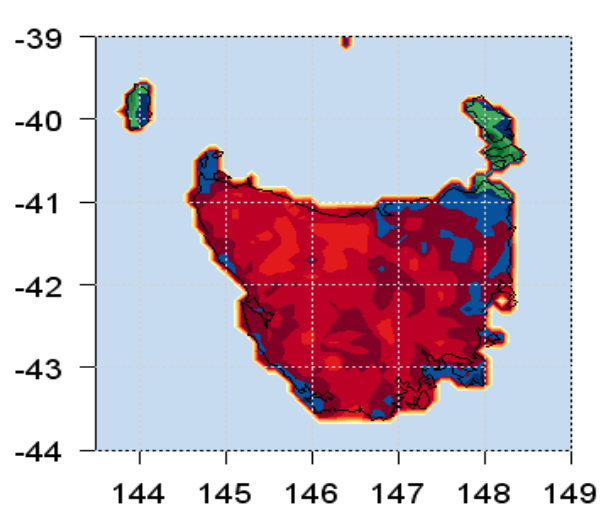

Figure 5. Average 500-yr RP of combined wind gust for 2080-2100 period based on six models using A2 scenario.
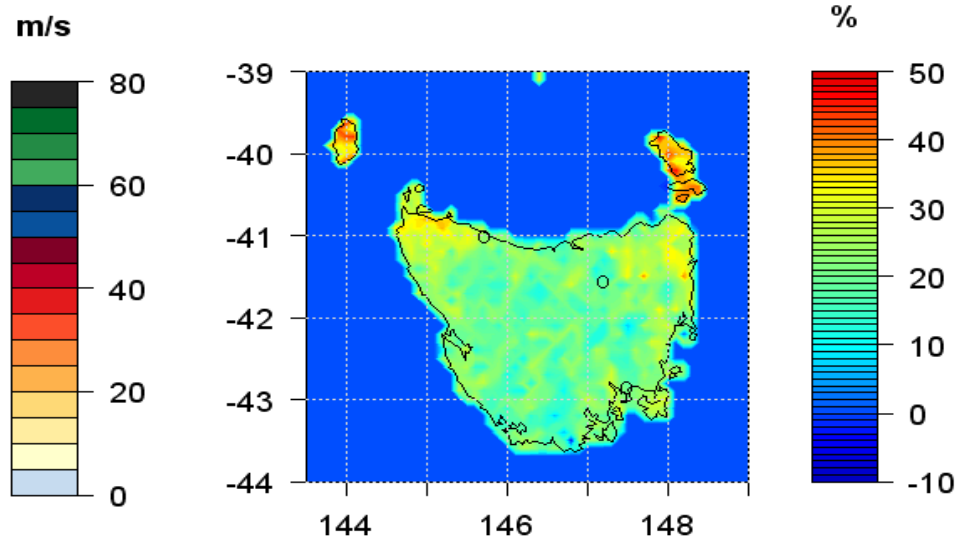

Figure 6. Wind hazard: \% increase by 2080-2100 w.r.t. current climate, A2 scenario.

In order to observe the impact of climate change on wind hazard at an observing station and compare with the current observed wind hazard, we consider the Launceston Airport recording station. Figure 8 shows the observed and CCAM modelled RPs of combined winds at Launceston Airport weather station (147.2E, 41.54S). The AS/NZ standard (AS/NZS 1170.2:2002) RP of wind speeds for Region A (wind loading standard) is also presented for comparison purposes. The CCAM-modelled RP of gust speeds for current climate (red dashed line) are higher than those observed especially at low RP. For wind hazard we are more interested in the high values of RP and they tend to be closer to the observed values.

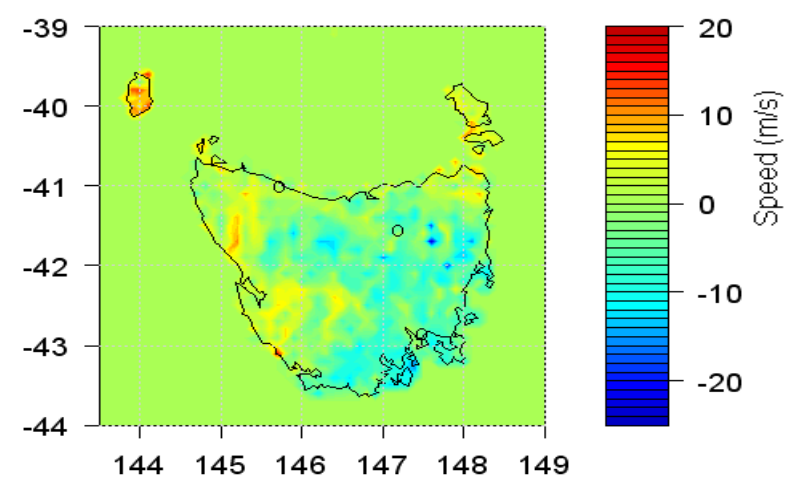

Figure 7. Wind hazard: \% difference between A2 and $\mathrm{B} 1$ for the $2080-2100$ period.

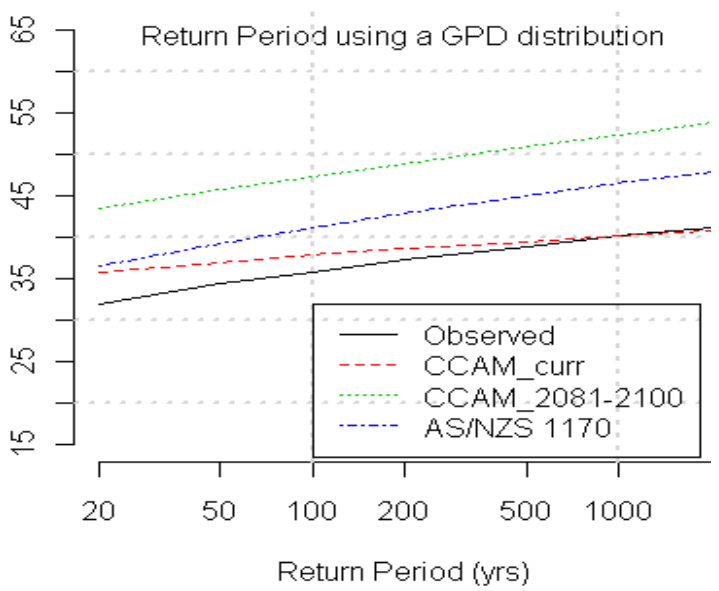

Figure 8. Launceston Airport observed and CCAM-modelled RP of combined wind speed.

The progressive increase of wind hazard with time (six model ensemble average) discussed above is presented here in graphical form; RP for 2081-2100 (green dotted line) are substantially higher than RP for current climate. It is interesting to observe the location of the return period for the wind loading standard curve. Based on the model ensemble average analysis we would be concerned that for the Launceston Airport Station the Australian/New Zealand standards for wind loading of structures is unlikely to remain a conservative estimate of the gust wind hazard well into the $21^{\text {st }}$ century. 


\section{DISCUSSION AND CONCLUSIONS}

A computational methodology developed for assessing severe wind hazard in the non-cyclonic regions of Australia has been presented. The methodology, initially developed for observational data, has also been applied to gridded data from climate models. This has enabled the regional study of wind hazard for both current climate and also for two future climate scenarios extending to the end of the $21^{\text {st }}$ century.

The methodology has been demonstrated by considering the current and future climate for Tasmania. Results from six climate models and two climate change emission scenarios (B1 \& A2) have been analysed. In general, the wind hazard increases in the Tasmanian region, however, the increase is not uniform across the state. The increase is most noticeable by the end of this century, especially in the coastal regions of the northeast and north-west and the Bass Strait Islands. This increase poses a challenge to the current Australian/NZ standard for wind loading of structures for some regions of Tasmania. However, current knowledge of the influence of climate change and the uncertain nature of the $21^{\text {st }}$ century trend for the ensemble average of the combined wind hazard (CSIRO, 2007) indicates that a cautious approach to codification is merited and further research on the impact of climate change on wind hazard is required.

\section{REFERENCES}

AS/NZS 1170:2:2011 (2011). Australian/New Zealand Standard. Structural design actions. Part 2: Wind actions.

Chen, K. (2004). Relative Risks Ratings for Local Government Areas. Risk Frontiers quarterly newsletter, Macquarie University, Australia. Vol. 3 issue 3, March 2004.

Coles, S. (2001). An Introduction to Statistical Modeling of Extreme Values. Springer series in statistics. London 2001.

Corney, S. Katzfey, J. McGregor, J. Grose, M. Bennett, J. White, C.J. Holz, G. and Bindoff, N.L. (2010). Climate Futures for Tasmania technical report: methods and results on climate modelling. Antarctic Climate and Ecosystems Cooperative Research Centre, Hobart, Tasmania.

CSIRO (2007). Climate Change in Australia - Wind Speed, Technical Report - Supplementary Material. http://www.climatechangeinaustralia.com.au/documents/resources/5 wind speed.pdf, accessed on 10/10/2011.

DOTARS -Department of Transport and Regional Services (2004). Natural Disasters in Australia: Reforming mitigation, relief and recovery arrangements.

Holmes, J.D. (2007), Wind Loading of Structures. Taylor \& Francis $2^{\text {nd }}$ Ed.

Middelmann, M. (2007). Natural Hazards in Australia. Identifying Risk and Analysis Requirements. Geoscience Australia. GeoCat \# 65444

Sanabria, L.A. and Cechet, R.P. (2007). A Statistical Model of Severe Winds. Geoscience Australia. GeoCat \# 65052.

Sanabria, L.A. and Cechet, R.P. (2010). Severe Wind Hazard Assessment using Monte Carlo Simulation. Journal of Environmental Modeling \& Assessment. Volume 15, Issue 2, (147-154).

Thomas, C. and Arthur, C. (2011). Modeling of Thunderstorm Hazard. Geoscience Australia Record (in preparation).

Trapp, R.J., Diffenbaugh, N.S., Brooks, H.E., Baldwin, M.E., Robinson, E.D., and Pal, J.S. (2007). Changes in severe thunderstorm environment during the $21^{\text {st }}$ century caused by anthropogenically enhanced global radiative forcing. Proc Nat. Acad. Sci. 104(50), pp 19719-19723.

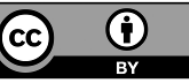

(C) Commonwealth of Australia (Geoscience Australia) 2011.

This publication is provided under a Creative Commons Attribution 3.0 Australia Licence with the permission of the CEO of Geoscience Australia. 